\title{
Development and validation of a prognostic score to predict mortality in patients with acute-on-chronic liver failure
}

\author{
Rajiv Jalan ${ }^{1, \dagger}$, Faouzi Saliba ${ }^{2,3, \dagger}$, Marco Pavesi $^{4, \dagger}$, Alex Amoros $^{4}$, Richard Moreau ${ }^{5}$, Pere Ginès ${ }^{6, *}$, \\ Eric Levesque ${ }^{2,3}$, Francois Durand ${ }^{5}$, Paolo Angeli ${ }^{7}$, Paolo Caraceni ${ }^{8}$, Corinna Hopf $^{9}$, \\ Carlo Alessandria ${ }^{10}$, Ezequiel Rodriguez ${ }^{6}$, Pablo Solis-Muñoz ${ }^{11}$, Wim Laleman ${ }^{12}$, Jonel Trebicka ${ }^{13}$, \\ Stefan Zeuzem ${ }^{14}$, Thierry Gustot ${ }^{15}$, Rajeshwar Mookerjee ${ }^{1}$, Laure Elkrief $^{5}$, German Soriano ${ }^{16}$, \\ Joan Cordoba ${ }^{17}$, Filippo Morando ${ }^{8}$, Alexander Gerbes ${ }^{9}$, Banwari Agarwal ${ }^{1}$, Didier Samuel ${ }^{2,3}$, \\ Mauro Bernardi ${ }^{8,18}$, Vicente Arroyo ${ }^{18}$, on behalf of the CANONIC study investigators \\ of the EASL-CLIF Consortium \\ ${ }^{1}$ Liver Failure Group, UCL Institute for Liver and Digestive Health, UCL Medical School, Royal Free Hospital, London, \\ United Kingdom; ${ }^{2}$ Hôpital Paul Brousse, Villejuif, France; ${ }^{3}$ Unité INSERM U785, Paris, France; ${ }^{4}$ Data Management Centre of the EASL \\ CLIF Consortium, Barcelona, Spain; ${ }^{5}$ INSERM U773 and Service d'Hépatologie, Hôpital Beaujon, Clichy, France; ${ }^{6}$ Hospital Clinic, Barcelona, \\ Spain; ${ }^{7}$ University of Padova, Italy; ${ }^{8}$ Policlinico St Orsola Malpighi, Bologna, Italy; ${ }^{9}$ Ludwig Maximilian University Munich, \\ Klinikum der Universität München, Germany; ${ }^{10}$ University of Turin, Italy; ${ }^{11}$ King's College Hospital, London, UK; ${ }^{12}$ University Hospital \\ Gasthuisberg, Leuven, Belgium; ${ }^{13}$ University Hospital Bonn, Germany; ${ }^{14} \mathrm{~J} . W$. Goethe University Hospital, Frankfurt, Germany; \\ ${ }^{15}$ Erasme Hospital Brussels, Belgium; ${ }^{16}$ Hospital de la Santa Creu i Sant Pau, Barcelona, Spain; ${ }^{17}$ Hospital Vall d'Hebron, Barcelona, \\ Spain; ${ }^{18}$ EASL-CLIF Consortium
}

\begin{abstract}
Background \& Aims: Acute-on-chronic liver failure (ACLF) is a frequent syndrome (30\% prevalence), characterized by acute decompensation of cirrhosis, organ failure(s) and high short-term mortality. This study develops and validates a specific prognostic score for ACLF patients.

Methods: Data from 1349 patients included in the CANONIC study were used. First, a simplified organ function scoring system (CLIF Consortium Organ Failure score, CLIF-C OFs) was developed to diagnose ACLF using data from all patients. Subsequently, in 275 patients with ACLF, CLIF-C OFs and two other independent predictors of mortality (age and white blood cell count) were combined to develop a specific prognostic score for ACLF (CLIF
\end{abstract}

Keywords: Acute-on-chronic liver failure; Cirrhosis; Multi-organ failure; Sepsis; Prognosis.

Received 21 January 2014; received in revised form 2 June 2014; accepted 11 June 2014; available online 17 June 2014

* Corresponding author. Address: Liver Unit, Hospital Clinic, University of Barcelona, Catalonia, Spain. Tel.: +34 93 2271713; fax: +34 932271779. E-mail address: PGINES@clinic.ub.es (P. Ginès).

$\dagger$ These authors contributed equally to this work.

$\star$ The complete list of CANONIC study investigators is reported at the end of the manuscript.

Abbreviations: ACLF, acute-on-chronic liver failure; AD, acute decompensation; CANONIC study, EASL-CLIF Acute oN chrONIC liver failure study; CLIF, chronic liver failure; CLIF-C ACLFs, CLIF Consortium ACLF score; CLIF-C OFs, CLIF Consortium organ failure score; CLIF-SOFAs, CLIF-sequential organ failure assessment score; CPs, Child-Pugh score; E, epinephrine; EASL, European Association for the Study of the Liver; $\mathrm{FiO}_{2}$, fraction of inspired oxygen; $\mathrm{HE}$, hepatic encephalopathy; INR, international normalized ratio; MAP, mean arterial pressure; MELD, model of end-stage liver disease; MELD-Nas, MELD-sodium score; NE, norepinephrine; $\mathrm{PaO}_{2}$, partial pressure of arterial oxygen; SOFA, sequential organ failure assessment; $\mathrm{SpO}_{2}$, pulse oximetric saturation.
Consortium ACLF score [CLIF-C ACLFs]). A concordance index (C-index) was used to compare the discrimination abilities of CLIF-C ACLF, MELD, MELD-sodium (MELD-Na), and Child-Pugh (CPs) scores. The CLIF-C ACLFs was validated in an external cohort and assessed for sequential use.

Results: The CLIF-C ACLFs showed a significantly higher predictive accuracy than MELDs, MELD-Nas, and CPs, reducing (19-28\%) the corresponding prediction error rates at all main time points after ACLF diagnosis (28, 90, 180, and 365 days) in both the CANONIC and the external validation cohort. CLIF-C ACLFs computed at $48 \mathrm{~h}, 3-7$ days, and 8-15 days after ACLF diagnosis predicted the 28 -day mortality significantly better than at diagnosis.

Conclusions: The CLIF-C ACLFs at ACLF diagnosis is superior to the MELDs and MELD-Nas in predicting mortality. The CLIF-C ACLFs is a clinically relevant, validated scoring system that can be used sequentially to stratify the risk of mortality in ACLF patients.

(C) 2014 European Association for the Study of the Liver. Published by Elsevier B.V. All rights reserved.

\section{Introduction}

Acute-on-chronic liver failure (ACLF) is a syndrome characterised by acute decompensation of cirrhosis, organ failure(s) and high short-term mortality [1], which was recently defined in the CANONIC study [2]. This was a large prospective observational investigation carried out in 29 European university hospitals. It included 1349 consecutive patients admitted with acute 
decompensation of cirrhosis (ascites, bacterial infection, gastro-intestinal haemorrhage, and hepatic encephalopathy) and followed-up for one year. The CANONIC study was organised in the setting of the European Association for the Study of the Liver - Chronic Liver Failure Consortium (EASL CLIF-C).

The results of the CANONIC study showed that ACLF occurs most frequently in relatively young individuals, affects approximately $30 \%$ of hospitalised cirrhotic patients, may develop in patients without previous decompensation, is associated with a 28 -day mortality rate of $33 \%$ ( $51 \%$ at 90 days) and is distinct from 'mere' decompensation of cirrhosis. ACLF is the most frequent indication for admission to the intensive care unit (ICU) [3]. In the US, about 200,000 patients with cirrhosis are hospitalised each year of which about 26,000 patients require ICU care $[1,3,4]$. An average ICU admission costs about $\$ 116,200$ and costs for the health care system are $\$ 3$ billion for cirrhotic patients requiring intensive care [3].

The diagnostic criteria for ACLF in the CANONIC study were based on the Chronic Liver Failure-SOFA score (CLIF-SOFAs), an adaptation for cirrhotic patients of the sepsis organ failure assessment score (SOFAs) widely used in the ICU [4]. The CLIF-SOFAs, however, is complex (based on 6 subscores, each with a 5-point range, assessing liver, kidney, brain, coagulation, respiration, and circulation), is based on consensus and expert opinion rather than data, and does not significantly improve the prediction accuracy of the model for end-stage liver disease (MELD) and MELDsodium (MELD-Na) scores [5-6].

The current study was aimed to simplify the original CLIF-SOFAs and develop a new score for ACLF patients (CLIF Consortium ACLF score, CLIF-C ACLFs) with a higher prognostic accuracy than the CLIF-SOFA, MELD, MELD-Na, and the Child-Pugh (CP) scores [7] for patients with ACLF. The study therefore had four main objectives. First, to develop a simpler and validated organ failure score (CLIF Consortium Organ Function score, CLIF-C OFs) for the diagnosis and grading of ACLF. Second, to design a more accurate prognostic score for ACLF patients (CLIF-C ACLFs), using the CLIF-C OFs and other prognostic clinical and biochemical data. Third, to compare the prognostic accuracy of the CLIF-C ACLFs to that of MELDs, MELD-Nas, and CPs. Fourth, to validate the prognostic accuracy of the CLIF-C ACLFs in an external prospective cohort of consecutive patients hospitalized in a single ICU and assess the score for sequential use.

The CANONIC study database was used as a derivation set for several reasons: first, it includes a large series of patients with acute decompensated cirrhosis and also with ACLF. Second, CANONIC patients were closely and prospectively followed-up for up to 1-year. Third, the population with ACLF included patients developing the syndrome either at study inclusion or during the hospitalization period. Finally, as patients were recruited from 29 centres in Europe, the CANONIC data are representative of the European patient population.

\section{Patient and methods}

Study populations

The study was performed in patients from three different populations. Both the derivation and the validation datasets came from studies approved by Ethical Review Boards of all study sites.

\section{JOURNAL OF HEPATOLOGY}

1. The CLIF-C OFs was developed using the baseline (i.e., enrolment) data of the whole CANONIC study population, which included 1349 patients (out of 2149 screened) admitted to 29-European hospitals within a period of 6 months for the treatment of decompensated cirrhosis. These patients were prospectively followed-up for one year [2]. In most patients (52\%) the aetiology of cirrhosis was alcoholic, in $19.5 \%$ it was associated with chronic hepatitis $C$ virus infection, and in $9.6 \%$ it was due to both alcohol and hepatitis C. In the remaining $18.9 \%$, cirrhosis was due to other causes. 345 patients (26.8\%) had no history of previous decompensation. Causes of hospitalization at study enrolment were ascites (66.8\%), hepatic encephalopathy (34.3\%), bacterial infections (24.2\%), and/or gastro-intestinal haemorrhage (16.4\%). 196 patients $(14.6 \%)$ were admitted to the ICU. The MELDs at enrolment in the whole series was 18.8 (SD: 7.5), and CPs was 9.7 (SD: 2.1).

2. The CLIF-C ACLFs was developed using data from 275 CANONIC patients with ACLF at enrolment, or those who developed ACLF within 28-days postenrolment. Diagnostic criteria for organ failures in the CANONIC study are described in Supplementary Table 1. The diagnosis of ACLF was based on the presence of at least renal failure or any other single organ failure if associated with renal dysfunction (serum creatinine $1.5-1.9 \mathrm{mg} / \mathrm{dl}$ ) and/or grade I-II hepatic encephalopathy (ACLF-1). Patients with two organ failures were graded as ACLF-2 and those with three or more organ failures as ACLF-3. At study enrolment, $70.1 \%$ of these patients were admitted to the ICU.

3. The external validation of the CLIF-C ACLFs was carried out using data from 225 ACLF patients consecutively admitted to the ICU at the Paul Brousse hospital, Villejuif, France [8]. Despite differences in the proportion of patients admitted to the ICU, this series of patients was selected as the validation set for the following reasons: (1) it was a prospective cohort; (2) all patients had the data needed for ACLF diagnosis and score calculations; (3) patients were followed-up for 90-days. Table 1 shows the clinical characteristics of patients included in the derivation and validation sets.

\section{Study outcomes}

The main study outcomes included all-cause mortality at 28, 90, 180, and 365 days after enrolment. All CANONIC patients were closely followed-up during the first 28 days. Subsequently, data on vital signs, causes of death and liver transplantation were obtained 3, 6, and 12 months after enrolment. The same information was collected for all ACLF patients in the validation cohort, who were followed-up for 3 months only. In both derivation and validation sets, there were no losses due to follow-up. At 90 days, 38/275 (13.8\%) ACLF patients underwent liver transplantation in the CANONIC dataset and 22/225 (9.8\%) in the validation cohort. One year after study enrolment, 53/275 (20\%) CANONIC patients with ACLF had been transplanted.

All the data required to compute CLIF-C ACLFs (as well as those used to compute MELDs, MELD-Nas, and CPs) were measured at the time of ACLF diagnosis (either at enrolment or within the 28-day post-enrolment follow-up). Patients' parameters were collected based on standard lab measurements performed at study enrolment and at day 2, 3-7, 8-14, 15-21, and 22-28 during the hospitalization. A central laboratory was not used for sample analyses. However, to assure the comparability of lab results, site labs were requested to use the same units and normal ranges; extensive remote monitoring and quality control were carried out during and after study termination.

Statistical methods

Variables used for the CLIF-C OFs and CLIF-C ACLFs were measured at enrolment and at ACLF diagnosis. The original CLIF-SOFAs included 6 subscores - one for each organ/system - each of them ranging from 0 to 4 (Supplementary Table 1). The 5 categories included in CLIF-SOFAs subscores and the corresponding cut-off values were derived from a consensus [2]. In the current study we assessed whether the cut-offs could be modified and/or if the number of the original categories of each subscore could be reduced maintaining the predictive ability of the aggregated score.

In univariate statistical comparisons, the $\chi^{2}$ test was used for categorical variables, Student's $t$ test and Mann-Whitney test for continuous variables. McNemar's test and paired $t$ test were used to compare repeated measurements of categorical and continuous variables, respectively. Proportional hazards models considering liver transplantation as a competing risk (PH-CR) were used to identify additional independent factors of mortality not included in the CLIF-C OFs system. Transplanted patients were considered as censored and the survival function was adjusted for the risk of liver transplantation at each study time point $[9,10]$. 


\section{Research Article}

Table 1. Baseline characteristics and outcome of patients with an acute-on-chronic liver failure (ACLF) episode included in the CANONIC study and in the external validation datasets.

\begin{tabular}{|c|c|c|c|}
\hline Patients' characteristics & $\begin{array}{l}\text { Derivation set } \\
\text { (CANONIC patients) } \\
(\mathrm{N}=275)\end{array}$ & $\begin{array}{l}\text { External validation set } \\
(\mathrm{N}=225)\end{array}$ & $p$ value \\
\hline Age (yr) & $54.5 \pm 12.1$ & $55.1 \pm 11.1$ & 0.567 \\
\hline Male sex & $176(64.0)$ & $171(76.0)$ & 0.005 \\
\hline Presence of ascites & $218(80.2)$ & $206(93.2)$ & $<0.001$ \\
\hline \multicolumn{4}{|l|}{ Etiology of cirrhosis } \\
\hline Alcohol & $147(54.7)$ & $158(70.2)$ & $<0.001$ \\
\hline $\mathrm{HCV}$ & $40(14.9)$ & $24(10.7)$ & 0.218 \\
\hline Alcohol + HCV & $29(10.8)$ & $14(6.2)$ & 0.100 \\
\hline \multicolumn{4}{|c|}{ Site of hospitalization at study enrolment } \\
\hline Intensive care unit & $192(70.1)$ & $225(100)$ & $<0.001$ \\
\hline \multicolumn{4}{|c|}{ Data used to compute CLIF-OF score } \\
\hline Serum bilirubin (mg/dl) & $12.3 \pm 11.5$ & $13.3 \pm 12.7$ & 0.360 \\
\hline Serum creatinine (mg/dl) & $2.0 \pm 1.3$ & $2.0 \pm 1.5$ & 0.721 \\
\hline HE grade I-II & $102(37.5)$ & $111(49.3)$ & \multirow[t]{2}{*}{$<0.001$} \\
\hline HE grade III-IV & $60(22.1)$ & $62(27.6)$ & \\
\hline INR & $2.2 \pm 1.0$ & $3.8 \pm 2.8$ & $<0.001$ \\
\hline Serum sodium (mEq/L) & $135 \pm 7$ & $134 \pm 8$ & 0.137 \\
\hline White-cell count $\left(\times 10^{9}\right.$ cells $\left./ \mathrm{L}\right)$ & $10.0 \pm 6.3$ & $7.8 \pm 4.3$ & $<0.001$ \\
\hline Use of vasopressors & $108(39.7)$ & $143(63.6)$ & $<0.001$ \\
\hline $\begin{array}{l}\mathrm{PaO}_{2} / \mathrm{FiO}_{2} 200-300 \\
\text { or } \\
\mathrm{SpO}_{2} / \mathrm{FiO}_{2} 214-357\end{array}$ & $71(25.8)$ & $93(41.3)$ & \multirow{2}{*}{$<0.001$} \\
\hline $\begin{array}{l}\mathrm{PaO}_{2} / \mathrm{FiO}_{2} \leq 200 \\
\text { or } \\
\mathrm{SpO}_{2} / \mathrm{FiO}_{2} \leq 214\end{array}$ & $39(14.2)$ & $86(38.2)$ & \\
\hline \multicolumn{4}{|c|}{ Acute-on-chronic liver failure (ACLF) grade and scores } \\
\hline ACLF grade 1 & $121(44.0)$ & $51(22.6)$ & \multirow{3}{*}{$<0.001$} \\
\hline ACLF grade 2 & $110(40.0)$ & $71(31.6)$ & \\
\hline ACLF grade 3 & $44(16.0)$ & $103(45.8)$ & \\
\hline Child-Pugh score & $11.1 \pm 2.0$ & $11.4 \pm 1.9$ & 0.104 \\
\hline MELD score & $28 \pm 8$ & $31 \pm 8$ & $<0.001$ \\
\hline MELD-Na score & $30 \pm 7$ & $33 \pm 7$ & $<0.001$ \\
\hline \multicolumn{4}{|l|}{ Mortality rates } \\
\hline 28-Day mortality & $93(33.8)$ & $117(52.0)$ & $<0.001$ \\
\hline 90-Day mortality & $133(48.4)$ & $141(62.7)$ & 0.002 \\
\hline 6-Month mortality & $143(52.0)$ & & \\
\hline 1-Year mortality & $159(57.8)$ & & \\
\hline
\end{tabular}

Data are numbers of patients $(\%)$ or mean \pm SD.

$\mathrm{HE}$, hepatic encephalopathy; $\mathrm{FiO}_{2}$, fraction of inspired oxygen; $\mathrm{PaO}_{2}$, partial pressure of arterial oxygen; $\mathrm{SpO}_{2}$, pulse oximetric saturation.

Baseline factors, not accounted for in the CLIF-C OFs, and significantly $(p<0.05)$ associated with mortality at $28,90,180$, and 365 days, were selected for the final models. PH-CR models including the CLIF-C OFs and all the other selected factors were fitted applying a forward step-wise selection method with $p$-in $=0.05$ and $p$-out $=0.1$. The factors that were independently associated with mortality at the main time points were included in the CLIF-C ACLFs. The coefficients estimated for each factor in the 28-day model, which provided the best predictive ability, were used as relative weights to compute the CLIF-C ACLFs.

The calibration of the CLIF-C ACLFs was assessed by comparing the actual observed risk and the average probability of dying at different time points predicted by the score. The Hosmer-Lemeshow test was used to assess the corresponding goodness-of-fit. The Harrell's concordance index (C-index) was used to assess the score's discrimination ability [11,12]. Since the CLIF-C ACLFs was derived based on a PH-CR model, C-index values and the corresponding 95\% confidence intervals (CIs) were estimated for each main study time point, treating the transplanted patients as censored at the end of the period, assuming that none of them would die before [9]. Statistical comparisons of the C-index between the CLIF-C ACLFs, CLIF-SOFAs, CLIF-C OFs, MELDs, MELD-Nas, and CPs were carried out for the main study time points using the integrated discriminating improvement (IDI) statistic [13].

For external validation [14], the CLIF-C ACLFs was computed with the validation data, and score performance was assessed and compared to that of the MELDs, MELD-Nas, and CPs by means of the same methods applied to the CANONIC data. To corroborate the results observed in the derivation and validation sets, a confirmatory analysis was carried out by estimating the Area Under the ROC curve (AUROC) of the CLIF-C ACLFs for predicting 28-day and 90-day mortality. This also allowed us to identify the best score cut-points, which maximized the sensitivity, specificity and predictive values.

The prognostic ability of the CLIF-C ACLFs, and MELDs for sequential use was assessed using a subset of CANONIC patients with ACLF and data available at the time of diagnosis and at $48 \mathrm{~h}, 3-7$ days, and at 8-15 days after diagnosis. Postdiagnosis $\mathrm{C}$-indexes were compared with the baseline by means of paired $t$ tests. 


\section{JOURNAL OF HEPATOLOGY}

Table 2. The CLIF-organ failure score system.

\begin{tabular}{|c|c|c|c|}
\hline Organ/system & Subscore $=1$ & Subscore $=2$ & Subscore $=3$ \\
\hline Liver & Bilirubin $<6 \mathrm{mg} / \mathrm{dl}$ & $\begin{array}{l}\text { Bilirubin } \geq 6 \mathrm{mg} / \mathrm{dl} \\
\text { and }<12 \mathrm{mg} / \mathrm{dl}\end{array}$ & Bilirubin $\geq 12 \mathrm{mg} / \mathrm{dl}$ \\
\hline Kidney & Creatinine $<2 \mathrm{mg} / \mathrm{dl}$ & $\begin{array}{l}\text { Creatinine } \geq 2 \mathrm{mg} / \mathrm{dl} \\
\text { and }<3.5 \mathrm{mg} / \mathrm{dl}\end{array}$ & $\begin{array}{l}\text { Creatinine } \geq 3.5 \mathrm{mg} / \mathrm{dl} \\
\text { or renal replacement }\end{array}$ \\
\hline Brain (West-Haven grade for $\mathrm{HE}^{\star}$ ) & Grade 0 & Grade 1-2 & Grade $3-4^{* *}$ \\
\hline Coagulation & INR $<2.0$ & INR $\geq 2.0$ and $<2.5$ & INR $\geq 2.5$ \\
\hline Circulatory & $\mathrm{MAP} \geq 70 \mathrm{mmHg}$ & MAP $<70 \mathrm{mmHg}$ & Use of vasopressors \\
\hline $\begin{array}{l}\text { Respiratory } \\
\mathrm{PaO}_{2} / \mathrm{FiO}_{2} \\
\text { or } \\
\mathrm{SpO}_{2} / \mathrm{FiO}_{2}\end{array}$ & $\begin{array}{l}>300 \\
\text { or } \\
>357\end{array}$ & $\begin{array}{l}\leq 300 \text { and }>200 \\
\text { or } \\
>214 \text { and } \leq 357\end{array}$ & $\begin{array}{l}\leq 200^{\#} \\
\text { or } \\
\leq 214^{\#}\end{array}$ \\
\hline
\end{tabular}

The shaded area describes criteria for diagnosing organ failures.

${ }^{*} \mathrm{HE}$, hepatic encephalopathy; $\mathrm{FiO}_{2}$, fraction of inspired oxygen; $\mathrm{PaO}_{2}$, partial pressure of arterial oxygen; $\mathrm{SpO}_{2}$, pulse oximetric saturation.

${ }^{* *}$ Patients submitted to Mechanical Ventilation $(\mathrm{MV})$ due to $\mathrm{HE}$ and not due to a respiratory failure were considered as presenting a cerebral failure $($ cerebral subscore $=3$ )

${ }^{\#}$ Other patients enroled in the study with MV were considered as presenting a respiratory failure (respiratory subscore $=3$ ).

\section{Results}

\section{ACLF study populations}

Table 1 shows the two ACLF study populations. ACLF patients in the external validation cohort were more frequently male and alcoholic, had more severe ACLF and higher rates of ascites, hepatic encephalopathy, respiratory and circulatory failure than ACLF patients in the CANONIC cohort. Consequently, the MELDs and MELD-Nas and the 28-day and 90-day mortality rates were higher in the validation cohort.

\section{Development of the CLIF-C OFs}

For each organ system, two new cut-points were chosen to distinguish three clinical severity categories that were directly correlated with the risk of dying at 28-days. The new cut-off values maximised the ability of the aggregated score (ranging 6 to 18 ) to predict 28-day mortality. Derivations of the resulting organ system subscores are reported in Table 2. The performance of the CLIF-C OFs (C-index: 0.72) in predicting 28-day mortality was identical to that of the original CLIF-SOFAs (C-index estimate: $0.72[p=0.856])$ and slightly but significantly superior to those of MELDs, MELD-Nas and CPs (C-index: 0.69 [ $p=0.015]$, $0.68[p=0.019]$ and $0.67[p=0.014]$ respectively).

\section{Development of the CLIF-C ACLFS}

Baseline factors, not included in the CLIF-C OFs and significantly associated with short- and long-term mortality, were alcoholic aetiology of cirrhosis, ascites (as clinically diagnosed), AST, serum sodium, serum potassium and white blood cell (WBC) count (Supplementary Table 2). Age was included in all models as a well-known potential confounder. Together with the CLIF-C OFs, age and log-transformed white blood cell count were selected as the best predictors. The CLIF-C ACLFs was computed by applying model coefficients and was trimmed between 0 and 100, since upper values did not modify the expected probabilities of dying by more than $1 \%$ :

CLIF-C ACLFs $=10 \times[0.33 \times$ CLIF-OFs $+0.04 \times$ Age +0.63

$$
\times \ln (\mathrm{WBC} \text { count })-2]
$$

The probability of death at time " $\mathrm{t}$ " can be estimated by the equation:

$P=1-e^{[-\mathrm{CI}(\mathrm{t}) \times \exp (\beta(\mathrm{t}) \times \mathrm{CLIF}-\mathrm{C} \text { ACLFs })}$

$\mathrm{CI}(\mathrm{t})$ and $\beta(\mathrm{t})$ are the cumulated baseline hazard and the score coefficient estimated by the model fitted for time $t$. At the main time points they are: $\mathrm{CI}(28)=0.0022, \beta(28)=0.0995 ; \mathrm{CI}(90)=$ $0.0079, \quad \beta(90)=0.0869 ; \quad \mathrm{CI}(180)=0.0115, \quad \beta(180)=0.0824$; $\mathrm{CI}(365)=0.0231, \beta(365)=0.0731$.

An online application to estimate the predicted death rate at time $t$ based on the CLIF-C ACLFs will be available at the CLIF Consortium website: http://www.clifconsortium.com/. A freely downloadable application is also available (CLIFC ACLFs).

\section{Calibration of the CLIF-C ACLFS}

Fig. 1 shows the observed and predicted probability of dying at 28 days by the CLIF-C ACLFs quintiles in the CANONIC and in the validation datasets. In the derivation set, predicted and observed probabilities of death were similar across the quintiles of CLIF-C ACLFs (Hosmer-Lemeshow $\chi^{2}=7.9, p=0.44$ ) and at the four study time points: 28-days: overall predicted 0.28 vs. overall observed 0.34; 90-days: 0.46 vs. 0.48 ; 6-month: 0.51 vs. 0.52; 1-year: 0.59 vs. 0.58 . The calibration of the CLIF-C ACLFs was also assessed for the validation dataset with similar results: observed and estimated probabilities of death were comparable at the main study time points (28-days: predicted $0.45 \mathrm{vs}$. observed 0.52 ; 90 days: $0.64 v$ s. 0.63 ) and the Hosmer-Lemeshow test for the 28-day mortality did not show a significant lack of fit $\left(\chi^{2}=4.0, p=0.26\right)$.

Discrimination ability of the CLIF-C ACLFs. Comparison with the CLIF-C OFs, CLIF-SOFAs, MELDs, MELD-Nas and the CPS

The C-index of the CLIF-C ACLFs for 28-day, 90-day, 6-month and 1 -year mortality $(0.76,0.73,0.72$, and 0.71$)$ was significantly better than those corresponding to the CLIF-C OFs $(0.72[p<0.001]$, 0.68 [ $p<0.001], 0.67[p<0.001]$, and 0.66 [ $p=0.003]$ ), and CLIFSOFAs $(0.72[p<0.001], 0.68[p<0.001], 0.67[p<0.001]$, and $0.66[p=0.002])$.

The CLIF-C ACLFs showed a significantly higher predictive discrimination than the MELDs, MELD-Nas, and CPs at 28-days and at the rest of the study time points (Table 3). The 


\section{Research Article}

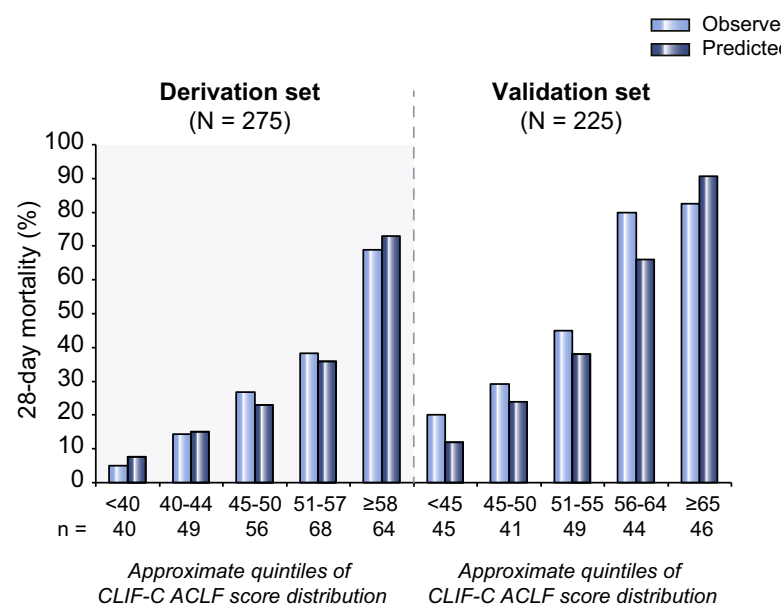

Fig. 1. Observed (light blue) vs. predicted (dark blue) 28-day mortality rates: according to the approximate quintiles of the CLIF-C ACLF score in the ACLF patients included in the derivation set (left side) and validation set (right side). The mortality probabilities predicted using the CLIF-C ACLFs score were similar to those observed in both sets of patients, thus indicating a good performance of the score throughout the whole range of CLIF-C ACLFs values.

absolute improvements of about 7-8 points in the C-index values with respect to the MELDs, MELD-Nas and CPs were consistently significant at all time points. Fig. 2 shows the corresponding percent improvement obtained with the CLIF-C ACLFs in prediction error rate with respect to the other scores (computed as percent reduction in discordance rate of the CLIF-C ACLFs vs. the reference (REF) score, i.e., $100 \times\left[C\right.$-index ${ }_{\text {LLIF-C }}$ ACLFs $-C$-index $\left.x_{\text {REF }}\right] /\left[1-\mathrm{C}\right.$-index $\left.\left.\mathrm{REF}_{\mathrm{REF}}\right]\right)$. The CLIF-C ACLFs consistently improved the prediction error rates observed for the MELDs, MELD-Nas, and CPs by $19 \%-28 \%$ at all study time points.

\section{Validation of the CLIF-C ACLFs}

The comparative $\mathrm{C}$-index estimates for the validation data cohort are shown in Table 3. The predictive ability of the CLIF-C ACLFs at

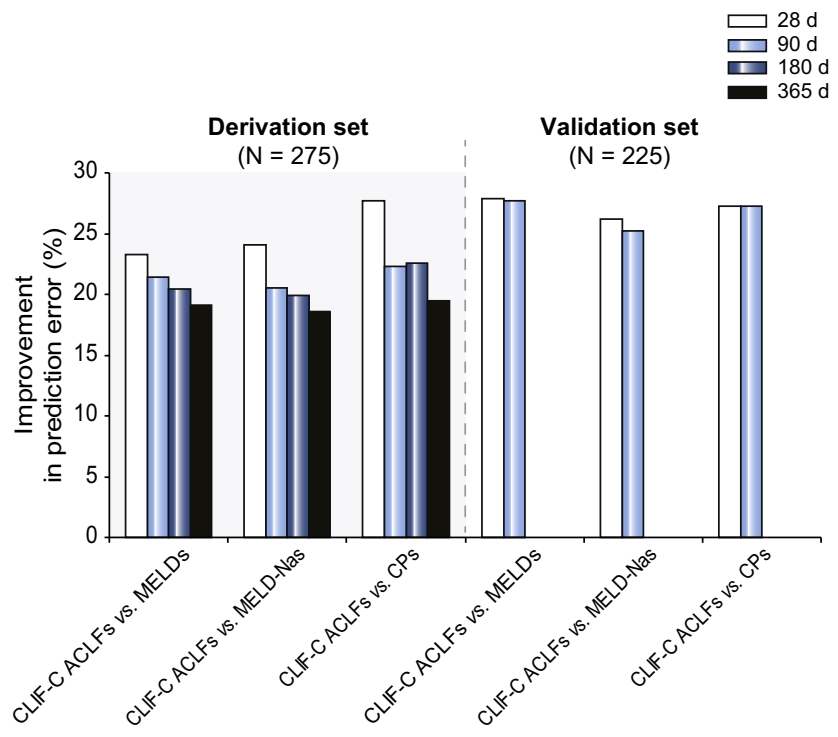

Fig. 2. Percent reduction in prediction error rates of the CLIF-C ACLF score as compared to the Child-Pugh, MELD, and MELD-Na scores at the main study time points. The prediction error rates observed using CLIF-C ACLFs were $19 \%$ to $28 \%$ lower than those observed for MELDs, MELD-Nas or CPs in the derivation set and $25 \%$ to $28 \%$ lower in the validation cohort.

each main time point was significantly better than for the MELDs, MELD-Nas and CPs (Supplementary Fig. 1). The CLIF-C ACLFs improved the 28-day and 90-day mortality predictions by about $25 \%$ to $28 \%$ as compared to the MELDs, MELD-Nas, and CPs (Fig. 2).

Figs. 3 and 4 show the results of the confirmatory analysis of the predictive ability of the CLIF-C ACLF score for the 28-day and 90-day mortality in the CANONIC patients with ACLF. As compared to the MELDs, MELD-Nas, and CPs, the AUROCs estimated for the CLIF-C ACLFs were significantly higher and indicated a $7 \%$ to $11 \%$ improvement in the discrimination ability, so confirming the concordance index estimates shown in Table 3. A CLIF-C ACLFs of 40 or lower had a $90 \%$ negative predictive value and

Table 3. Predictive discrimination ability of the CLIF-C ACLF score as compared with the MELDs and MELD-Nas. ACLF patients in the CANONIC database and in the validation dataset.

\begin{tabular}{|c|c|c|c|c|}
\hline & $\begin{array}{l}\text { CLIF-C ACLF score } \\
\text { C-index }(95 \% \mathrm{CI})\end{array}$ & $\begin{array}{l}\text { Child-Pugh score } \\
\text { C-index }(95 \% \mathrm{Cl})\end{array}$ & $\begin{array}{l}\text { MELD score } \\
\text { C-index }(95 \% \mathrm{Cl})\end{array}$ & $\begin{array}{l}\text { MELD-Na score } \\
\text { C-index }(95 \% \mathrm{Cl})\end{array}$ \\
\hline \multicolumn{5}{|c|}{ CANONIC PATIENTS (N = 275) } \\
\hline 28-day mortality & $0.760(0.715-0.805)$ & $0.668(0.610-0.726)$ & $0.687(0.635-0.738)$ & $0.684(0.632-0.736)$ \\
\hline$p$ value vs. CLIF-C* & & $<0.001$ & $<0.001$ & $<0.001$ \\
\hline 90-day mortality & $0.732(0.691-0.773)$ & $0.655(0.605-0.705)$ & $0.659(0.615-0.710)$ & $0.663(0.617-0.709)$ \\
\hline$p$ value vs. CLIF-C* & & $<0.001$ & $<0.001$ & 0.001 \\
\hline 180-day mortality & $0.723(0.683-0.763)$ & $0.642(0.593-0.691)$ & $0.652(0.607-0.697)$ & $0.654(0.609-0.699)$ \\
\hline$p$ value vs. CLIF-C* & & $<0.001$ & $<0.001$ & 0.001 \\
\hline 365-day mortality & $0.707(0.668-0.746)$ & $0.636(0.588-0.683)$ & $0.638(0.595-0.682)$ & $0.640(0.597-0.683)$ \\
\hline$p$ value vs. CLIF-C* & & $<0.001$ & $<0.001$ & $<0.001$ \\
\hline \multicolumn{5}{|c|}{ Validation database $(\mathrm{N}=225)$} \\
\hline 28-day mortality & $0.744(0 \cdot 702-0.787)$ & $0.653(0.603-0.704)$ & $0.645(0.593-0.697)$ & $0.648(0.597-0.700)$ \\
\hline$p$ value vs. CLIF-C* & & $<0.001$ & $<0.001$ & $<0.001$ \\
\hline 90-day mortality & $0.736(0.696-0.776)$ & $0.647(0.599-0.695)$ & $0.635(0.585-0.684)$ & $0.637(0.588-0.686)$ \\
\hline$p$ value vs. CLIF-C* & & $<0.001$ & $<0.001$ & $<0.001$ \\
\hline
\end{tabular}

${ }^{*} p$ values from the Integrated Discriminating Improvement (IDI) statistics test. 


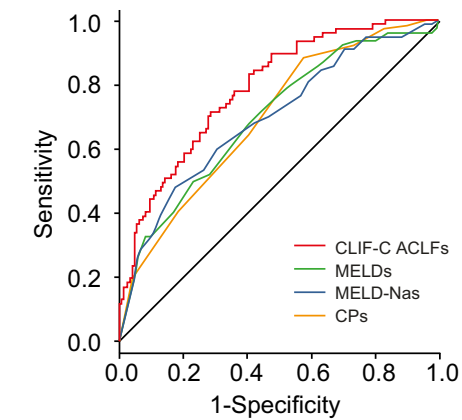

\begin{tabular}{llll}
\hline & & $\begin{array}{l}\text { AUROC } \\
(95 \% \mathrm{Cl})\end{array}$ & $\begin{array}{l}p \text { value } \\
\text { vs. CLIF-C ACLF }\end{array}$ \\
\hline- & CLIF-C ACLFs & $0.79(0.73-0.85)$ & \\
- & MELDs & $0.70(0.62-0.77)$ & 0.0089 \\
- & MELD-Nas & $0.70(0.62-0.77)$ & 0.0097 \\
\hline & CPs & $0.70(0.63-0.77)$ & 0.0075 \\
\hline
\end{tabular}

Fig. 3. Accuracy of the CLIF-C ACLFs as compared to that of MELDs, MELD-Nas and CPs in predicting 28-day mortality of the CANONIC patients with ACLF. Comparison of the area under the ROC curves (AUROCs) estimated for each score. The CLIF-C ACLFs showed a significantly higher predictive ability in comparison with all the other scores. (This figure appears in colour on the web.)

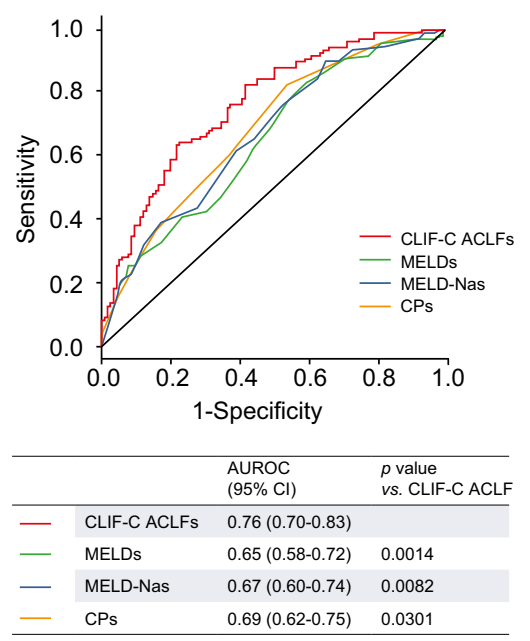

Fig. 4. Accuracy of the CLIF-C ACLFs as compared to that of MELDs, MELD-Nas, and CPs in predicting 90-day mortality of the CANONIC patients with ACLF. Comparison of the area under the ROC curves (AUROCs) estimated for each score. The CLIF-C ACLFs showed a significantly higher predictive ability in comparison with all the other scores. (This figure appears in colour on the web.)

$97 \%$ sensitivity, while a score of 60 or higher allowed for a $82 \%$ positive predictive value and $94 \%$ specificity. A score cut-off of 51 maximized both the sensitivity (64\%) and the specificity (75\%), but was found to have lower negative (69\%) and positive (70\%) predictive values. In the validation set comparable results were observed: a CLIF-C ACLFs of 40 or lower had a $77 \%$ negative predictive value and $97 \%$ sensitivity, while a score of 60 or higher showed a $91 \%$ positive predictive value and $93 \%$ specificity.

\section{Sequential use of the CLIF-C ACLFS}

Table 4 reports the C-index estimates for the CLIF-C ACLFs and the MELDs computed at ACLF diagnosis and at $48 \mathrm{~h}, 3-7$ days, and $8-15$ days after ACLF diagnosis in the 256 CANONIC study

\section{JOURNAL OF HEPATOLOGY}

patients with follow-up clinical and laboratory data. Both scores, when used sequentially, improved their predictive performance. The CLIF-C ACLFs computed at 3-7 days and 8-15 days after ACLF diagnosis predicted 28-day and 90-day mortality significantly better than the CLIF-C ACLFs at ACLF diagnosis. Interestingly, the sequence of $\mathrm{C}$-index estimates reflected a consistent difference of 7-8 points between the CLIF-C ACLFs and the MELDs in the corresponding discrimination abilities.

\section{Discussion}

The observation in the CANONIC study that ACLF occurs in $30 \%$ of hospitalised cirrhotic patients, is associated with a 28-day mortality rate of $32 \%$, has well-defined diagnostic criteria, and is pathophysiologically related to systemic inflammation provides the rationale for new investigations on the mechanism and management of this syndrome $[1,2,8,15,16]$. Consequently, it is necessary to develop an accurate prognostic score for ACLF patients.

Any specific score for ACLF should have the following characteristics. First, the score should be as simple as possible to facilitate clinical use and be based on the function of the vital organ-systems defining the ACLF syndrome [2]. Second, parameters estimating organ failure should be easy to obtain in standard hospitalization settings $[3,8,15,16]$. Third, the cut-off levels for the definition of organ system failure should have prognostic significance. Fourth, the score should provide computed figures, estimating risk of mortality but also allow an easy stratification of patients with clinical and prognostic significance. Finally, it should clearly improve the predictive ability of the main prognostic scores currently available (MELDs and CPs).

The first step of this study was to develop a simplified score based on organ function (the CLIF-C OFs), using the data obtained from all patients included in the CANONIC study [2]. As indicated above, the main objective of this new score was to diagnose and grade the severity of ACLF. The CLIF-C OFs, which has a 3-point range per organ system and an aggregated score ranging from 6 to 18 , is simpler than the original CLIF-SOFAs used in the CANONIC study from which it derives (5 point ranges and aggregated score range 0-24) and accomplishes all the above mentioned criteria except the last one. Interestingly, the prognostic significance of cut-off levels for the diagnosis of organ failures of the original CLIF-SOFAs were confirmed in the CLIF-C OFs [2]. Therefore, diagnosis of organ failure and the stratification of ACLF proposed by the CANONIC study are also applicable using the CLIF-C OFs. In the CANONIC patients with ACLF, the predictive accuracy of the CLIF-C OFs was identical to that of the CLIF-SOFA score and slightly but significantly better than those of the MELDs, MELD-Nas, and CPs.

The second step of the study was to develop a prognostic score better than the CLIF-C OFs for patients with ACLF. The strategy used was to analyse patients with ACLF in the CANONIC database looking for independent factors associated with mortality not included in the CLIF-OFs and to subsequently fit a final survival model with the CLIF-OFs and the selected factors. Age and white blood cell count (a crude marker of systemic inflammation) were found to be the best predictors. These parameters have also been found to be independently associated with mortality in previous studies [2,8,15-19]. Although in the CANONIC study, white blood cell count was higher in patients with alcoholic liver 
Table 4. Sequential use of the CLIF-C ACLF and MELD score to predict mortality in CANONIC patients with ACLF and post-enrolment clinical and laboratory follow-up.

\begin{tabular}{|c|c|c|c|c|c|c|c|c|}
\hline & \multicolumn{4}{|c|}{ 28-day mortality } & \multicolumn{4}{|c|}{ 90-day mortality } \\
\hline & \multicolumn{2}{|c|}{ CLIF-C ACLF score } & \multicolumn{2}{|c|}{ MELD score } & \multicolumn{2}{|c|}{ CLIF-C ACLF score } & \multicolumn{2}{|c|}{ MELD score } \\
\hline & $\begin{array}{l}\text { C-index } \\
(95 \% \mathrm{Cl})\end{array}$ & $\begin{array}{l}p \text { value vs. } \\
\text { baseline }\end{array}$ & $\begin{array}{l}\text { C-index } \\
(95 \% \mathrm{Cl})\end{array}$ & $\begin{array}{l}p \text { value vs. } \\
\text { baseline }\end{array}$ & $\begin{array}{l}\text { C-index } \\
(95 \% \mathrm{Cl})\end{array}$ & $\begin{array}{l}p \text { value vs. } \\
\text { baseline }\end{array}$ & $\begin{array}{l}\text { C-index } \\
(95 \% \mathrm{Cl})\end{array}$ & $\begin{array}{l}p \text { value vs. } \\
\text { baseline }\end{array}$ \\
\hline $\begin{array}{l}\text { CLIF-C-ACLF score } \\
\text { at enrolment }(\mathrm{N}=256)\end{array}$ & $\begin{array}{l}0.751 \\
(0.701-0.800)\end{array}$ & & $\begin{array}{l}0.679 \\
(0.621-0.737)\end{array}$ & & $\begin{array}{l}0.712 \\
(0.666-0.759)\end{array}$ & & $\begin{array}{l}0.653 \\
(0.602-0.703)\end{array}$ & \\
\hline $\begin{array}{l}\text { CLIF-C-ACLF score } \\
\text { at } 48 \text { hours }(N=186)\end{array}$ & $\begin{array}{l}0.801 \\
(0.747-0.854)\end{array}$ & 0.0956 & $\begin{array}{l}0.721 \\
(0.658-0.783)\end{array}$ & 0.1890 & $\begin{array}{l}0.751 \\
(0.700-0.802)\end{array}$ & 0.1336 & $\begin{array}{l}0.680 \\
(0.625-0.736)\end{array}$ & 0.3312 \\
\hline $\begin{array}{l}\text { CLIF-C-ACLF score } \\
\text { at 3-7 days }(N=189)\end{array}$ & $\begin{array}{l}0.822 \\
(0.767-0.877)\end{array}$ & 0.0179 & $\begin{array}{l}0.749 \\
(0.682-0.815)\end{array}$ & 0.0389 & $\begin{array}{l}0.774 \\
(0.722-0.827)\end{array}$ & 0.0217 & $\begin{array}{l}0.706 \\
(0.646-0.765)\end{array}$ & 0.0824 \\
\hline $\begin{array}{l}\text { CLIF-C-ACLF score } \\
\text { at } 8-15 \text { days }(N=154)\end{array}$ & $\begin{array}{l}0.866 \\
(0.809-0.923)\end{array}$ & 0.0001 & $\begin{array}{l}0.799 \\
(0.729-0.870)\end{array}$ & 0.0008 & $\begin{array}{l}0.790 \\
(0.733-0.847)\end{array}$ & 0.0072 & $\begin{array}{l}0.710 \\
(0.643-0.776)\end{array}$ & 0.0958 \\
\hline
\end{tabular}

${ }^{*} p$ value $v s$. CLIF-C score at enrolment.

disease and active alcoholism, the data also showed that irrespective of aetiology, higher white blood cell count was associated with more severe grades of ACLF [2]. Through the final model including a CLIF-C OFs with these two new variables, we were able to obtain a new score (CLIF-C ACLFs) ranging between 0 and 100 points, which predicted the risk of mortality accurately.

The concordance index analysis in the derivation cohort showed that the CLIF-C ACLFs was more accurate in predicting short-term and long-term mortality than their predecessors CLIF-SOFAs and CLIF-C OFs (data not shown) and also than MELDs, MELD-Nas, and CPs. The differences in predictive discrimination between the CLIF-C ACLFs and MELDs, MELD-Nas, and CPs at 28 and 90-days were even more significant in the validation cohort. The fact that these patients were more severely ill than those in the derivation cohort probably accounts for this observation. The analysis of the prediction errors, observed with the CLIFC ACLFs compared with MELDs, MELD-Nas, and CPs in both the derivation and the validation cohorts, is additional evidence of the superiority of the CLIF-C ACLFs over the other scores in predicting mortality in ACLF patients. Using the CLIF-C ACLFs leads to a substantial improvement (19\% to $28 \%$ ) in the discrimination ability observed with MELDs, MELD-Nas, and CPs.

We also assessed whether the CLIF-C ACLF score could be useful if applied sequentially to identify response to an intervention, deterioration despite intervention and perhaps act as a guide to determine whether further interventions are likely to be futile. The data showed that the performance of the CLIF-C ACLFs improved significantly if repeated $48 \mathrm{~h}, 3-7 \mathrm{~d}$ or $8-15 \mathrm{~d}$ after the initial determination. These data suggest that, in clinical practice, the CLIF-C ACLFs, like the SOFAs, which is widely used in the ICU for non-cirrhotic patients, can be updated on a daily basis providing additional prognostic information [4]. Currently, no validated evidence-based tools guide the decision-making. Sequential measurements of the CLIF-C ACLFs may help to define potential futility.

In summary, using the CANONIC database we derived a new, evidence-based and simpler organ-failure score (CLIF-C OFs) to diagnose organ failures and ACLF in cirrhotic patients. The CLIF-C OFs includes parameters most commonly used in the management of ACLF patients and shows a prognostic accuracy similar to its predecessor, the CLIF-SOFAs but significantly higher than MELDs, MELD-Nas, and CPs. Combining the CLIF-C OFs with age and white blood cell count, the CLIF-C ACLFs was obtained as a specific prognostic score for ACLF patients and validated in an external cohort of ACLF patients. CLIF-C ACLFs allowed a significant improvement of the discrimination ability as compared to the MELDs, MELD-Nas, and CPs, as indicated by a $19 \%$ to $28 \%$ reduction in percent prediction errors observed in both the derivation and validation datasets. The CLIF-C ACLFs also proved to be potentially useful for sequential use after ACLF diagnosis but this will require further validation.

\section{Financial support}

The CLIF Consortium is supported by an unrestricted grant from Grifols. Rajiv Jalan is supported by a comprehensive biomedical research centre, UK grant, Richard Moreau was supported by an INSERM-APHP fellowship and Jonel Trebicka by grants from the Deutsche Forschungsgemeinschaft (SFB TRR57 project 18).

\section{Conflict of interest}

\section{The EASL-CLIF Consortium}

The EASL-CLIF Consortium is a network of 63 European university hospitals, aimed at stimulating research on pathophysiology, diagnostic and treatment on Chronic Liver Failure. During the period 2009-2012 the EASL-CLIF Consortium had received unrestricted grants from Grifols and Gambro. Grifols has prolonged its unrestricted grant for an additional period of four years. There is no other support for the Consortium. The Fundació Clinic, a foundation ruled by the Hospital Clinic and University of Barcelona, administers the EASL-CLIF Consortium grants. V. Arroyo (Chairman), M. Bernardi (Vice-Chairman), and members of the Steering Committee have no relationship with Grifols or Gambro other than conferences at international meetings (from which they may receive an honorarium) or as investigators on specific projects unrelated to the consortium. Up to now the EASL-CLIF Consortium has not performed any study promoted by pharmaceutical companies. The scientific agenda of the EASL-CLIF Consortium and the specific research protocols are made exclusively by the Steering Committee members without any participation of pharmaceutical companies.

\section{Individual authors}

R. Jalan received research funding from Vital Therapies, has served on the Scientific Advisory Board for Conatus Pharma, 
received lecture fees from Gambro, has on-going research collaboration with Gambro, Grifols, and is the Principal Investigator of an industry sponsored study (Sequana Medical). He is also the inventor of a drug, L-ornithine phenylacetate, which UCL has licensed to Ocera Therapeutics. F. Saliba has received speaker honorarium and/or grant research from Novartis, Astellas, Roche, Merck Sharp \& Dohme, Pfizer, Gambro and Vital Therapies. P. Ginès has received speaker honorarium and research funding from Grifols, served on the scientific advisory board for Ferring and Sequana and received research funding from Sequana. J. Cordoba has served as a consultant to Ocera. A. Gerbes has served as a consultant to CSL Behring. S. Zeuzem has served as a consultant to Abbott, Achillion, AstraZeneca, BMS, Boehringer Ingelheim, Gilead, Janssen Cilag, Merck, Novartis, Presidio, Roche, Santaris, Vertex. V. Arroyo has received grant and research support from Grifols. All other authors declare that they have no conflicts of interest.

\section{Authors' contributions}

R. Jalan, R. Moreau, M. Pavesi, A. Amoros, F. Saliba, M. Bernardi, and V. Arroyo participated in the writing group. R. Moreau, R. Jalan, P. Ginès, M. Pavesi, F. Durand, T. Gustot, J. Trebicka, and $\mathrm{V}$. Arroyo designed the CANONIC study with input from all other authors. R. Moreau, R. Jalan, M. Pavesi, A. Amoros, F. Saliba, and V. Arroyo participated in data analysis and interpretation. F. Durand, P. Angeli, P. Caraceni, C. Hopf, C. Alessandria, E. Rodríguez, P. Solis-Muñoz, W. Laleman, J. Trebicka, S. Zeuzem, T. Gustot, R. Mookerjee, L. Elkrief, G. Soriano, J. Cordoba, F. Morando, A. Gerbes, B. Agarwal, and D. Samuel participated in patient recruitment and data collection along with investigators of the CANONIC study. F. Saliba, E. Levesque, and D. Samuel provided the data from Paul-Brousse Hospital for external validation. V. Arroyo was responsible for obtaining funding and overall project collaboration.

\section{CANONIC study investigators of the EASL-CLIF Consortium (alphabetical order)}

1. Patricia Aguilar Melero, Hospital Universitario Reina Sofía. Centro de Investigación Biomédica en Red Enfermedades Hepáticas y Digestivas (CIBERehd). Instituto Maimónides de Investigación Biomédica de Córdoba (IMIBIC), Córdoba, Spain.

2. Agustin Albillos, Servicio de Gastroenterología, Hospital Universitario Ramón y Cajal, Universidad de Alcalá, Centro de Investigación Biomédica en Red Enfermedades Hepáticas y Digestivas (CIBERehd), Madrid, Spain.

3. Rafael Bañares, Hospital General Universitario Gregorio Marañón, Instituto de Investigación Sanitaria Gregorio Marañón (IiSGM), Centro de Investigación Biomédica en Red Enfermedades Hepáticas y Digestivas (CIBERehd), School of Medicine, Universidad Complutense, Madrid, Spain.

4. Daniel Benten, Department of Gastroenterology and Hepatology, University Medical Center HamburgEppendorf, Hamburg, Germany.

5. Massimo Bocci, Department of Gastroenterology and Hepato-Pancreatology, Erasme Hospital, Université Libre de Bruxelles, Brussels, Belgium.

\section{JOURNAL OF HEPATOLOGY}

6. Paolo Caraceni, Semeiotica Medica, Policlinico S. OrsolaMalpighi Department of Medical and Surgical Sciences University of Bologna, Bologna, Italy.

7. María-Vega Catalina, Hospital General Universitario Gregorio Marañón, Instituto de Investigación Sanitaria Gregorio Marañón (IiSGM), Centro de Investigación Biomédica en Red Enfermedades Hepáticas y Digestivas (CIBERehd), Madrid, Spain.

8. Jun Liong Chin, Liver Unit, St Vincent's University Hospital, Dublin, Ireland.

9. Minneke J. Coenraad, Leiden University Medical Centre, Department of Gastroenterology-Hepatology, Leiden, The Netherlands.

10. Mar Concepción, Department of Gastroenterology, Hospital de la Santa Creu i Sant Pau, Barcelona, Universitat Autònoma de Barcelona, Barcelona, Spain.

11. Audrey Coilly, Centre Hépato-Biliaire, Hôpital Paul-Brousse, Assistance Publique-Hôpitaux de Paris, Villejuif, France.

12. Livia Dorn, Department of Gastroenterology and Hepatology, Innsbruck Medical University, Innsbruck, Austria.

13. Carme Deulofeu, Data Management Centre, CLIF Consortium, Hospital Clinic, Barcelona, Spain.

14. Laure Elkrief, Service d'Hépatologie, Hôpital Beaujon, Assistance Publique Hôpitaux de Paris, Clichy; Inserm U773, Centre de Recherche Biomédicale Bichat-Beaujon CRB3, Clichy and Paris; and Université Paris Diderot-Paris 7, Paris, France.

15. Javier Fernandez, Liver Unit, Hospital Clinic, University of Barcelona, CLIF Consortium Centro de Investigación Biomédica en Red Enfermedades Hepáticas y Digestivas (CIBERehd), Barcelona, Spain.

16. Elisabet Garcia, Data Management Centre, CLIF Consortium, Hospital Clinic, Barcelona, Spain.

17. Angelo Gatta, Department of Medicine, University of Padova, Padova, Italy.

18. Ludmila Gerber, Department of Medicine I, JW Goethe University Hospital, Frankfurt, Germany.

19. Andrea de Gottardi; University Clinic of Visceral Surgery and Medicine of Berne, Berne, Switzerland.

20. Henning Grønbæk, Department of Medicine V, Unit of Hepatology and Gastroenterology, Aarhus University Hospital, Aarhus, Denmark.

21. Monica Guevara, Liver Unit, Hospital Clinic, University of Barcelona, Centro de Investigación Biomédica en Red Enfermedades Hepáticas y Digestivas (CIBERehd), Barcelona, Spain.

22. AnneKristin Hausen, Department of Internal Medicine I, University Hospital of Bonn, Bonn, Germany.

23. Corinna Hopf, Liver Center Munich, Department of Medicine 2, Klinikum der LMU München-Grosshadern, Munich, Germany.

24. Stine Karlsen, Department of Medicine V, Unit of Hepatology and Gastroenterology, Aarhus University Hospital, Aarhus, Denmark.

25. Ansgar W. Lohse Department of Gastroenterology and Hepatology, University Medical Center Hamburg-Eppendorf, Hamburg, Germany.

26. Caterina Maggioli, Semeiotica Medica, Policlinico S. OrsolaMalpighi Department of Medical and Surgical Sciences University of Bologna, Bologna, Italy. 
27. Daniel Markwardt, Liver Center Munich, Department of Medicine 2, Klinikum der LMU München-Grosshadern, Munich, Germany.

28. Javier Martinez, Servicio de Gastroenterología, Hospital Universitario Ramón y Cajal, Madrid, Spain.

29. Alfredo Marzano, Division of Gastroenterology and Hepatology, San Giovanni Battista Hospital, University of Turin, Turin, Italy.

30. Manuel de la Mata García, Hospital Universitario Reina Sofía. Centro de Investigación Biomédica en Red Enfermedades Hepáticas y Digestivas (CIBERehd). Instituto Maimónides de Investigación Biomédica de Córdoba (IMIBIC), Córdoba, Spain.

31. P. Aiden McCormick, Liver Unit, St Vincent's University Hospital, Dublin, Ireland.

32. Francisco Mesonero, Servicio de Gastroenterología, Hospital Universitario Ramón y Cajal, Madrid, Spain.

33. José Luis Montero Álvarez, Hospital Universitario Reina Sofía. Centro de Investigación Biomédica en Red Enfermedades Hepáticas y Digestivas (CIBERehd). Instituto Maimónides de Investigación Biomédica de Córdoba (IMIBIC), Córdoba, Spain.

34. Rajeshwar P Mookerjee, Institute of Liver and Digestive Health, Liver Failure Group, Royal Free Campus, London, United Kingdom.

35. Filippo Morando, Department of Medicine, University of Padova, Padova, Italy.

36. Christophe Moreno, Department of Gastroenterology and Hepato-Pancreatology, Erasme Hospital, Université Libre de Bruxelles, Brussels, Belgium.

37. Bernhard Morrell; University Clinic of Visceral Surgery and Medicine of Berne, Berne, Switzerland.

38. Christian Mortensen, Department of Gastroenterology, Hvidovre Hospital, University of Copenhagen, Copenhagen, Denmark.

39. Frederik Nevens, Department of Liver and Biliopancreatic Diseases, University Hospital Gasthuisberg, KU Leuven, Leuven, Belgium.

40. Markus Peck-Radosavljevic, Department of Gastroenterology and Hepatology, Medical University of Vienna, Vienna, Austria.

41. Gustavo Pereira, Liver Unit, Hospital Clinic, University of Barcelona, Centro de Investigación Biomédica en Red Enfermedades Hepáticas y Digestivas (CIBERehd), Barcelona, Spain.

42. Alessandro Risso, Division of Gastroenterology and Hepatology, San Giovanni Battista Hospital, University of Turin, Turin, Italy.

43. Mario Rizzetto; Division of Gastroenterology and Hepatology, San Giovanni Battista Hospital, University of Turin, Turin, Italy.

44. Ezequiel Rodriguez, Liver Unit, Hospital Clinic, University of Barcelona, Centro de Investigación Biomédica en Red Enfermedades Hepáticas y Digestivas (CIBERehd), Barcelona, Spain.

45. Antonietta Romano, Department of Medicine, University of Padova, Padova, Italy.

46. Didier Samuel, Centre Hépato-Biliaire, Hôpital Paul Brousse, Assistance Publique-Hôpitaux de Paris, Villejuif, France.
47. Tilman Sauerbruch, Department of Internal Medicine I, University Hospital of Bonn, Bonn Germany.

48. Macarena Simon-Talero, Servicio de Hepatologia, Hospital Vall d'Hebron, Universitat Autònoma de Barcelona, Centro de Investigación Biomédica en Red Enfermedades Hepáticas y Digestivas (CIBERehd), Barcelona, Spain.

49. Pablo Solis-Muñoz, Institute of Liver Studies and the Cellular Biology of Inflammation, King's College London, London, United Kingdom.

50. German Soriano, Department of Gastroenterology, Hospital de la Santa Creu i Sant Pau, Barcelona, Universitat Autònoma de Barcelona, Centro de Investigación Biomédica en Red Enfermedades Hepáticas y Digestivas (CIBERehd), Instituto de Salud Carlos III, Barcelona, Spain.

51. Jan Sperl, Department of Hepatogastroenterology, Institute for Clinical and Experimental Medicine, Prague, Czech Republic.

52. Walter Spindelboeck, Division of Gastroenterology and Hepatology, Department of Internal Medicine, Medical University of Graz, Graz, Austria.

53. Rudolf Stauber, Division of Gastroenterology and Hepatology, Department of Internal Medicine, Medical University of Graz, Graz, Austria.

54. Christian Steib, Liver Center Munich, Department of Medicine 2, Klinikum der LMU München-Grosshadern, Munich, Germany.

55. Dominique Valla, Service d'Hépatologie, Hôpital Beaujon, Assistance Publique Hôpitaux de Paris, Clichy; Inserm U773, Centre de Recherche Biomédicale Bichat-Beaujon CRB3, Clichy and Paris; and Université Paris Diderot-Paris 7, Paris, France.

56. Len Verbeke, Department of Liver and Biliopancreatic Diseases, University Hospital Gasthuisberg, KU Leuven, Leuven, Belgium.

57. Hans Van Vlierberghe, Department of Gastroenterology and Hepatology, Ghent University Hospital, Ghent, Belgium.

58. Wolfgang Vogel, Department of Gastroenterology and Hepatology, Innsbruck Medical University, Innsbruck, Austria.

59. Henning Wege, Department of Gastroenterology and Hepatology, University Medical Center HamburgEppendorf, Hamburg, Germany.

60. Tania Welzel, Department of Medicine I, JW Goethe University Hospital, Frankfurt, Germany.

61. Chris Willars, Liver Intensive Care Unit, King's College Hospital, London, United Kingdom.

62. Maria Yago Baenas, Institute of Liver and Digestive Health, Liver Failure Group, Royal Free Campus, London, United Kindgom.

63. Giacomo Zaccherini, Semeiotica Medica, Policlinico S. Orsola-Malpighi Department of Medical and Surgical Sciences University of Bologna, Bologna, Italy.

\section{Supplementary data}

Supplementary data associated with this article can be found, in the online version, at http://dx.doi.org/10.1016/j.jhep.2014. 06.012 . 


\section{JOURNAL OF HEPATOLOGY}

\section{References}

[1] Jalan R, Gines P, Olson JC, et al. Acute-on chronic liver failure. J Hepatol 2012;57:1336-1348.

[2] Moreau R, Jalan R, Gines P, et al. Acute-on-chronic liver failure is a distinct syndrome developing in patients with acute decompensation in cirrhosis. Gastroenterology 2013;144:1426-1437.

[3] Olson JC, Wendon JA, Kramer DJ, et al. Intensive care of the patient with cirrhosis. Hepatology 2011;54:1864-1872.

[4] Ferreira FL, Bota DP, Bross A, Melot C, Vincent JL. Serial evaluation of the SOFA score to predict outcome in critically ill patients. JAMA 2001;286: 1754-1758.

[5] Kamath PS, Kim WR. The model for end-stage liver disease (MELD) Hepatology 2007;45:797-805.

[6] Kim WR, Biggins SW, Kremers WK, et al. Hyponatremia and mortality among patients on the liver-transplant waiting list. N Engl J Med 2008;359: 1018-1026.

[7] Pugh RN, Murray-Lyon IM, Dawson JL, Pietroni MC, Williams R. Transection of the oesophagus for bleeding oesophageal varices. Br J Surg 1973;60: 646-649.

[8] Levesque E, Hoti E, Azoulay D, et al. Prospective evaluation of the prognostic scores for cirrhotic patients admitted to an intensive care unit. J Hepatol 2012;56:95-102.

[9] Fine JP, Gray RJ. A proportional hazards model for the subdistribution of a competing risk. J Am Stat Assoc 1999;94:496-509.

[10] Wolbers M, Koller MT, Witteman JC, Steyerberg EW. Prognostic models with competing risks. Methods and application to coronary risk prediction. Epidemiology 2009;20:555-561.
[11] Harrell Jr FE, Lee KL, Mark DB. Multivariable prognostic models: issues in developing models, evaluating assumptions and adequacy, and measuring and reducing errors. Stat Med 1996;15:361-387.

[12] Pencina MJ, D'Agostino RB. Overall $C$ as a measure of discrimination in survival analysis: model specific population value and confidence interval estimation. Stat Med 2004;23:2109-2123.

[13] Pencina MJ, D’Agostino Sr RB, D’Agostino Jr RB, Vasan RS. Evaluating the added predictive ability of a new marker: from area under the ROC curve to reclassification and beyond. Stat Med 2008;27:157-172.

[14] Altman DG, Royston P. What do we mean by validating a prognostic model? Stat Med 2000;19:453-473.

[15] Shawcross DL, Sharifi Y, Canavan JB, et al. Infection and systemic inflammation, not ammonia, are associated with Grade 3/4 hepatic encephalopathy, but not mortality in cirrhosis. J Hepatol 2011;54:640-649.

[16] Jalan R, Stadlbauer V, Sen S, Cheshire L, Chang YM, Mookerjee RP. Role of predisposition, injury, response and organ failure in the prognosis of patients with acute-on-chronic liver failure: a prospective cohort study. Crit Care 2012;16:R227.

[17] O'Grady JG, Alexander GJ, Hayllar KM, Williams R. Early indicators of prognosis in fulminant hepatic failure. Gastroenterology 1989;97:439-445.

[18] Cazzaniga M, Dionigi E, Gobbo G, Fioretti A, Monti V, Salerno F. The systemic inflammatory response syndrome in cirrhotic patients: relationship with their in-hospital outcome. J Hepatol 2009;51:475-482.

[19] Cholongitas E, Marelli L, Shusang V, et al. A systematic review of the performance of the model for end-stage liver disease (MELD) in the setting of liver transplantation. Liver Transpl 2006;12:1049-1061. 